\title{
Estimating the Prevalence of Sleep-Disordered Breathing Among Collegiate Football Players
}

\author{
Devon A Dobrosielski PhD, Dalton Nichols, Julia Ford, Amy Watts, \\ Joshua N Wilder MSc ATC CSCS, and Tamara Douglass-Burton MSc RRT
}

\begin{abstract}
BACKGROUND: Obstructive sleep apnea is a clinical disorder characterized by loud snoring, apneic episodes, and chronic sleep disruption. Collegiate football players exhibit several risk factors for OSA, including large neck circumference and high body mass index, although the prevalence of OSA in this cohort is unknown. METHODS: The STOP-BANG questionnaire was administered at random to members of a collegiate football team and used to stratify the players into high and low risk for sleep-disordered breathing (SDB). Those who completed the questionnaire were then evaluated for SDB during preseason camp using a single-channel (finger pulse oximetry) photoplethysmography-based device. SDB was defined as an apnea-hypopnea index of $\geq 5$. RESULTS: Of 56 players who underwent overnight photoplethysmography monitoring, valid results were available for 51. Forty-eight percent of the players were high-risk (neck size $=44.6 \pm 2.2 \mathrm{~cm}$, body mass index $=33.0 \pm 5.4$ ) versus low-risk (neck size $=41.4 \pm 2.8 \mathrm{~cm}$, body mass index $=27.6 \pm 3.6$ ) (both $P$ values <.001). An apnea-hypopnea index of $\geq 5$ was found in $2(8.3 \%, 95 \%$ CI $1.0-20.0 \%)$ high-risk and $2(7.7,95 \%$ CI 1.0-18.4\%) low-risk players. Two offensive linemen, a linebacker, and a tight end accounted for the positive cases. CONCLUSIONS: Based on our sample, we estimate the prevalence of SDB among collegiate football players to be $8 \%$, regardless of risk stratification. Given the strong link between SDB and cardiovascular disease, these data underscore the importance of screening and subsequent treatment of SDB in this highly conditioned yet potentially vulnerable group of athletes. Key words: photoplethysmography; athletes; sleep disorders; STOP-BANG; dual energy $x$-ray absorptiometry (DXA). [Respir Care 2016;61(9):1144-1150. (C) 2016 Daedalus Enterprises]
\end{abstract}

\section{Introduction}

Obstructive sleep apnea (OSA) is a clinical disorder characterized by loud snoring, apneic episodes (cessation

Dr Dobrosielski, Mr Nichols, Ms Ford, and Ms Watts are affiliated with the Department of Kinesiology; Mr Wilder is affiliated with the Department of Athletics; Ms Douglass-Burton is affiliated with the Department of Interprofessional Health Studies; and Dr Dobrosielski and Mr Wilder are affiliated with the Towson Research Academy for Collaborative Sport Science (TRACS), Towson University, Towson, Maryland.

Ms Ford presented a version of this paper at the Mid-Atlantic Chapter of the American College of Sports Medicine Annual Meeting, held October 31 to November 1, 2014, in Harrisburg, Pennsylvania. Ms DouglasBurton presented a version of this paper at the Maryland Sleep Society Sixth Annual Conference, held April 24 and 25, 2015, in Towson, Maryland and presented a version of this paper as an Editors' Choice abstract at the AARC Congress 2015, held November 7-10, 2015, in Tampa, Florida. Ms Watts presented a version of this paper at the Mid-Atlantic of breathing) and chronic sleep disruption. ${ }^{1}$ The estimated prevalence of OSA among United States adults is $4 \%,{ }^{2}$ and prospective studies have demonstrated that OSA has dire effects on cardiovascular health..$^{2,3}$ Although OSA affects primarily middle to older age adults, it can occur in younger individuals. ${ }^{4}$ American football players represent a unique cohort of young adults who are well-conditioned athletes but have several risk factors for OSA, including

Athletic Trainers Association Annual Meeting, May 29-31, 2015, in Virginia Beach, Virginia.

This research was supported by a grant from the Towson Academy of Scholars. The authors have disclosed no conflicts of interest.

Correspondence: Devon A Dobrosielski PhD, Department of Kinesiology, Towson University, 8000 York Road, Towson, MD 21252. E-mail: ddobrosielski@towson.edu.

DOI: $10.4187 /$ respcare. 04520 
male sex, high body mass index, and a large neck circumference. Indeed, others ${ }^{5,6}$ have demonstrated that OSA, or sleep-disordered breathing (SDB), is present in approximately $14-19 \%$ of professional football players. This represents an approximately 5-fold higher prevalence compared with young community-based volunteers, ${ }^{7}$ yet thousands of young men of similar size play football at the collegiate level and are likely to go undiagnosed. Since alertness, reaction time, and concentration are linked to proper sleep, ${ }^{8}$ those with SDB may experience detriments in athletic as well as academic performance. Importantly, given the strong association between SDB and the development of hypertension, ${ }^{2}$ these athletes may suffer from greater cardiovascular disease burden. Although treatment of SDB will undoubtedly minimize cardiovascular disease risk, the presence of this disorder among collegiate football players must first be recognized.

Accordingly, the purpose of the present investigation was to estimate the prevalence of SDB in collegiate football players. A secondary aim was to evaluate the relationship between markers of SDB and body composition parameters using state-of-the-art dual-energy x-ray absorptiometry imaging. We hypothesized that the prevalence of SDB among collegiate football players would be similar to that observed in professional players and that total body and regional body fat would be higher among those who exhibit SDB compared with those without the disorder.

\section{Methods}

\section{Study Participants}

The study sample was randomly selected from the 100man roster of the Towson University Division 1AA collegiate football team. Testing took place during a preseason mini-camp in August 2014. The Towson University Institutional Review Board approved the study protocol, and each participant provided written informed consent. All players were eligible to participate. Individuals were excluded if they had previously been diagnosed with a sleep disorder.

Participants completed a STOP-BANG questionnaire and Epworth Sleepiness Scale to assess risk for SDB and level of daytime sleepiness, respectively. Players were given a Morpheus Ox (WideMed Ltd., Herziliya, Israel) portable single-channel (pulse oximeter), photoplethysmography (PPG)-based sleep-monitoring device to be worn for one night. An athletic trainer provided instructions for home use. Players were encouraged not to alter sleep patterns. Within 3 weeks of sleep monitoring, body composition was assessed using dual-energy $\mathrm{x}$-ray absorptiometry.

\section{QUICK LOOK}

\section{Current knowledge}

Sleep-disordered breathing (SDB) is a common disorder associated with obesity that has dire consequences on cardiovascular health. The prevalence of SDB among professional football players is approximately $14-19 \%$, which represents an approximately 5 -fold higher prevalence compared with young, community-based volunteers. Thousands of young men of similar size play football at the collegiate level, yet the prevalence of SDB in this cohort has not been studied.

\section{What this paper contributes to our knowledge}

Using overnight photoplethysmography, we found the estimated prevalence of SDB in collegiate football players to be $8 \%$, regardless of risk stratification. Thirtyfive percent of the players reported a clinically important degree of daytime sleepiness. Greater total body and central fat, as assessed by dual-energy $\mathrm{x}$-ray absorptiometry, was observed in those with greater oxygen desaturation index.

\section{STOP-BANG Questionnaire}

The STOP-BANG questionnaire was utilized to provide risk stratification for SDB. ${ }^{9}$ This questionnaire consists of 8 items that address the following risk factors: (1) snoring, (2) daily fatigue, (3) someone observing you to stop breathing during sleep, (4) high blood pressure, (5) high body mass index $\left(>35 \mathrm{~kg} / \mathrm{m}^{2}\right),(6)$ age $>50 \mathrm{y}$, (7) large neck circumference $(>40 \mathrm{~cm})$, and (8) male sex. Neck circumference was measured around the thickest portion of the neck by an investigator. High risk for SDB was defined as $\geq 3$ affirmative answers to the 8 STOP-BANG items.

\section{Epworth Sleepiness Scale}

The Epworth Sleepiness Scale (ESS) is a validated 8-item questionnaire that measures subjective sleepiness. ${ }^{10}$ Participants were asked to rate how likely they are to fall asleep in the following situations: (1) sitting and reading, (2) watching television, (3) sitting inactive in a public place, (4) being a passenger in a car for at least $1 \mathrm{~h},(5)$ lying down to rest in the afternoon, (6) sitting and talking to someone, (7) sitting quietly after lunch, and (8) driving a car stopped in traffic. Each question is scored from 0 to 3. ESS values range from 0 (unlikely to fall asleep in any situation) to 24 (high chance of falling asleep in all 8 situations). High risk for daytime sleepiness was defined as an ESS total score $>10$. 


\section{Morpheus Ox Sleep Monitoring Device}

The theory behind the use of the Morpheus Ox device to obtain PPG-derived signals is described elsewhere. ${ }^{11}$ Briefly, PPG uses a pulse oximeter to detect blood volume changes in the microvascular bed of the fingertip, and proprietary software ${ }^{12,13}$ is used to analyze the PPG for baseline variations, envelope, and rate. These parameters are combined to generate a PPG-derived respiration waveform that is correlated with saturation reductions to detect a clinically relevant apnea-hypopnea index (AHI). In this study, we report a 4\% (2007 American Academy of Sleep Medicine criteria) oxygen desaturation index (ODI4) and 3\% ODI (ODI3) (2012 American Academy of Sleep Medicine criteria) for defining hypopneas. Furthermore, the PPG software platform facilitates the detection and measurement of respiratory events, sleep/wake epochs, and total sleep time. ${ }^{12,13}$ Respiratory events occurring during sleep epochs are averaged over total sleep time to generate the AHI value. Data were excluded from analysis if the PPG estimated sleep time was $<2 \mathrm{~h}$.

\section{Body Composition}

Height and weight were measured in metric units using a stadiometer and digital scale, respectively. Total body dual-energy x-ray absorptiometry scans were acquired in the frontal plane using the Lunar iDXA scanner (GE Healthcare, Madison, Wisconsin). Abdominal visceral fat was computed over the android region and reported in $\mathrm{kg}$. The android region is roughly $10 \mathrm{~cm}$ in height, extending from the iliac crest toward the head, a height that is $20 \%$ of the distance from the iliac crest to the base of the mandible. Lunar iDXA scans were analyzed with the enCORE software, version 14.0 (GE Healthcare, Madison, Wisconsin).

\section{Statistical Analysis}

All data are reported as mean SD. Independent-samples $t$ tests were used to compare demographic and body composition parameters between players classified as high-risk versus low-risk based on responses to the STOP-BANG questionnaire. Confidence intervals are reported for SDB prevalence estimates. A $\phi$ coefficient of association was calculated to examine the differences between risk categories in the number of players with an ESS $>10$ and ODI $\geq 5$ events/h. Independent-samples $t$ tests were conducted to examine differences in body composition parameters between players who exhibited sleep parameters (AHI, ODI 4 , and ODI3) of $\geq 5$ events/h versus those below this threshold. The limit for statistical significance was set at $P<.05$. Data analysis was conducted using Stata 10.1 (StataCorp, College Station, Texas).

\section{Results}

Of 56 players who underwent overnight PPG monitoring, valid results were available for 51 . The reason for insufficient data was that the Morpheus Ox device estimated sleep time of $<2 \mathrm{~h}$. The remaining sample consisted of 13 linebackers (25\%), 10 offensive linemen (20\%), 9 defensive linemen (18\%), and 3 tight ends (6\%). The remaining $31 \%$ of the cohort consisted of defensive backs, wide receivers, kickers, quarterbacks, and a long snapper. Seven linebackers, 6 offensive linemen, 8 defensive linemen, and one tight end were classified as high-risk for OSA, whereas only one wide receiver, a quarterback, and the long snapper were classified as high-risk based on the STOP-BANG questionnaire. Table 1 displays participant characteristics according to risk category. Fifty-six percent of the sample were African-American. The players classified as high-risk weighed more $(115.5 \pm 18.2 \mathrm{~kg}$ vs $95.6 \pm 17.2 \mathrm{~kg}, P<.001)$ and had a larger neck circumference $(44.6 \pm 2.2 \mathrm{~cm}$ vs $41.4 \pm 2.8 \mathrm{~cm}, P<.001)$ and higher body mass index $\left(33.0 \pm 5.4 \mathrm{~kg} / \mathrm{m}^{2}\right.$ vs $27.6 \pm 3.6$ $\left.\mathrm{kg} / \mathrm{m}^{2}, P<.001\right)$ compared with the low-risk players.

Body composition parameters from the dual-energy $\mathrm{x}$ ray absorptiometry scan are given in Table 2. Scheduling conflicts resulted in 4 players being unavailable for dualenergy x-ray absorptiometry testing. As expected, due to differences in weight, high-risk players had greater total lean mass $(82.0 \pm 7.9 \mathrm{~kg}$ vs $73.3 \pm 8.4 \mathrm{~kg}, P<.001)$, total fat mass $(26.5 \pm 11.9 \mathrm{~kg}$ vs $20.1 \pm 10.0 \mathrm{~kg}, P=.043)$ and trunk fat mass $(12.6 \pm 6.7 \mathrm{~kg}$ vs $9.1 \pm 5.3 \mathrm{~kg}, P=.047)$. The large girth of several of the players required that we complete half-body scans, thereby precluding the software from estimating visceral fat in 6 players. No differences in visceral fat were observed between the high- and low-risk groups.

Regarding PPG-derived sleep parameters, total sleep time was low but did not differ between the high- and low-risk groups $(4.2 \pm 1.1 \mathrm{~h}$ vs $3.8 \pm 0.9 \mathrm{~h}, P=.17)$. An AHI between 5 and 15 events/h was found in $2(8.3 \%$, 95\% CI 1.0-20.0\%) high-risk and 2 (7.7, 95\%, CI $1.0-$ $18.4 \%)$ low-risk players. Two offensive linemen (AHI $=7$ and 11 events/h), a linebacker $(\mathrm{AHI}=11$ events/h), and a tight end (AHI $=9$ events/h) accounted for the positive cases. No players were observed to have moderate to severe OSA, defined as having an AHI $>15$. Of the 4 positive cases, 3 players had an ESS score $>10$, a scoring threshold suggestive of a clinically important degree of daytime sleepiness. Thus, the estimated prevalence of an AHI between 5 and 15 events/h among those at high risk for daytime sleepiness was $15.7 \%$ (95\% CI $1.0-33.1 \%$ ). However, we found no significant difference between risk categories in the number of players who reported an ESS score $>10$ (high risk: 8 [44\%] vs low risk: 10 [56\%]; $\phi=0.02, P=.90)$. Similarly, we found no significant 
Table 1. Characteristics of Players per STOP-BANG-Derived Risk Category

\begin{tabular}{|c|c|c|c|c|c|c|c|}
\hline & \multicolumn{3}{|c|}{ High-Risk Players $(n=25)$} & \multicolumn{3}{|c|}{ Low-Risk Players $(n=26)$} & \multirow{2}{*}{$P^{*}$} \\
\hline & Mean \pm SD & Range & Median & Mean $\pm \mathrm{SD}$ & Range & Median & \\
\hline Age, y & $19.8 \pm 1.4$ & $18-23$ & 19.5 & $19.4 \pm 1.4$ & $17-22$ & 19.0 & .38 \\
\hline Height, cm & $187.5 \pm 9.4$ & $157-201$ & 188.0 & $185.7 \pm 6.6$ & $170-198$ & 185.4 & .44 \\
\hline Weight, kg & $115.5 \pm 18.2$ & $79-143$ & 111.6 & $95.6 \pm 17.2$ & $76-135$ & 93.9 & $<.001$ \\
\hline BMI, $\mathrm{kg} / \mathrm{m}^{2}$ & $33.0 \pm 5.4$ & $24.3-45.9$ & 32.2 & $27.6 \pm 3.6$ & $22.7-34.4$ & 26.6 & $<.001$ \\
\hline Neck circumference, $\mathrm{cm}$ & $44.6 \pm 2.2$ & $40.0-49.0$ & 45 & $41.4 \pm 2.8$ & $38.0-49.0$ & 41.0 & $<.001$ \\
\hline ESS total score & $9.3 \pm 4.2$ & $1-16$ & 9 & $8.4 \pm 3.7$ & $0-16$ & 9 & .40 \\
\hline Ethnicity & \multicolumn{3}{|c|}{$n(\%)$} & \multicolumn{3}{|c|}{$n(\%)$} & \\
\hline African-American & \multicolumn{3}{|c|}{$16(31)$} & \multicolumn{3}{|c|}{$13(25)$} & \\
\hline White & \multicolumn{3}{|c|}{$6(12)$} & \multicolumn{3}{|c|}{$13(25)$} & \\
\hline Mixed race & \multicolumn{3}{|c|}{$1(2.3)$} & \multicolumn{3}{|c|}{$1(2.3)$} & \\
\hline Hispanic & \multicolumn{3}{|c|}{$1(2.3)$} & \multicolumn{3}{|c|}{$0(0)$} & \\
\hline $\begin{array}{l}\text { * Equality of variances not assume } \\
\text { BMI = body mass index } \\
\text { ESS = Epworth Sleepiness Scale }\end{array}$ & & & & & & & \\
\hline
\end{tabular}

Table 2. Body Composition per STOP-BANG-Derived Risk Category

\begin{tabular}{|c|c|c|c|c|c|c|c|}
\hline & \multicolumn{3}{|c|}{ High Risk $(n=22)$} & \multicolumn{3}{|c|}{ Low Risk $(n=25)$} & \multirow{2}{*}{$P^{*}$} \\
\hline & Mean \pm SD & Range & Median & Mean \pm SD & Range & Median & \\
\hline Total mass, $\mathrm{kg}^{* *}$ & $112.9 \pm 16.4$ & $77.5-138.2$ & 112.9 & $97.3 \pm 16.8$ & $76.5-130.6$ & 95.1 & .002 \\
\hline Total fat mass, $\mathrm{kg}$ & $26.5 \pm 11.9$ & $6.8-44.7$ & 22.6 & $20.1 \pm 10.0$ & $7.6-43.7$ & 15.4 & .043 \\
\hline Total fat, $\%$ & $22.6 \pm 7.9$ & $8.8-34.3$ & 21.1 & $19.8 \pm 6.8$ & $9.7-33.4$ & 17.4 & .16 \\
\hline Total lean mass, $\mathrm{kg}$ & $82.0 \pm 7.9$ & $66.5-103.5$ & 82.1 & $73.3 \pm 8.4$ & $58.9-89.1$ & 75.3 & $<.001$ \\
\hline Trunk fat mass, kg & $12.6 \pm 6.7$ & $2.2-25.3$ & 10.8 & $9.1 \pm 5.3$ & $2.3-19.5$ & 6.6 & .047 \\
\hline Trunk fat $\%$ of total fat mass & $45.5 \pm 6.3$ & $31.9-56.8$ & 45.1 & $43.5 \pm 6.5$ & $27.0-56.7$ & 43.9 & .23 \\
\hline Abdominal visceral fat mass, $\mathrm{kg} \dagger$ & $0.42 \pm 0.4$ & $0.04-1.1$ & 0.25 & $0.30 \pm 0.3$ & $0.00-1.3$ & 0.20 & .24 \\
\hline Visceral fat $\%$ of total fat mass $\dagger$ & $1.7 \pm 1.1$ & $0.39-4.2$ & 1.5 & $1.5 \pm 1.2$ & $0.01-4.8$ & 1.2 & .47 \\
\hline \multicolumn{8}{|c|}{$\begin{array}{l}\text { * Equality of variances not assumed. } \\
* * \text { Total mass }=\text { total fat mass }+ \text { total lean mass }+ \text { total bone mass (not displayed). } \\
\dagger \text { Data unavailable for } 4 \text { high-risk and } 2 \text { low-risk players. }\end{array}$} \\
\hline
\end{tabular}

difference between risk categories in the number of players with an ODI4 or ODI3 of $\geq 5$ events/h (high risk: 4 [67\%] versus low risk: 2 [33\%]; $\phi=0.153, P=.28$ and high risk: 8 [53\%] versus low risk: 7 [47\%]; $\phi=0.096$, $P=.50$, respectively).

Body composition parameters in the players who exhibited SDB were compared with those of players without SDB. Whereas players with SDB tended to have higher total fat mass (SDB: $31.8 \pm 9.5 \mathrm{~kg}$ vs $21.2 \pm 11.2 \mathrm{~kg}$, $P=.12)$, trunk fat mass (SDB: $15.1 \pm 5.0 \mathrm{~kg}$ vs nonSDB: $10.1 \pm 6.2 \mathrm{~kg}, P=.14)$, abdominal visceral fat mass (SDB: $0.73 \pm 0.43 \mathrm{~kg}$ vs non-SDB: $0.31 \pm 0.33 \mathrm{~kg}$, $P=.24$ ), and total body fat (SDB: $26.7 \pm 4.9 \%$ vs nonSDB: $20.3 \pm 7.5 \%, P=.07)$, the data were not statistically significant. These analyses were repeated using ODI as a surrogate for AHI. Table 3 displays data according to an ODI4 threshold of 5 . Of note, players with ODI4 $\geq 5$ had greater total body mass $(P=.03)$, whereas differences in total fat mass $(P=.062)$, total body fat percentage $(P=.069)$, and trunk fat mass $(P=.08)$ approached statistical significance. Similar trends were observed when an ODI3 $\geq 5$ events/h was used as a cut-point (data not shown).

\section{Discussion}

Two previous studies have evaluated SDB among football players. George et al ${ }^{14}$ stratified 302 players from the National Football League (NFL) into high and low risk for SDB using the Multivariable Apnea Prediction index. Fifty-two players (high-risk $=38$; low-risk $=14$ ) subse- 
Table 3. Body Composition Parameters According to 4\% Oxygen Desaturation Index

\begin{tabular}{|c|c|c|c|c|c|c|c|}
\hline & \multicolumn{3}{|c|}{ ODI4 $<5$ events/h $(n=41)$} & \multicolumn{3}{|c|}{ ODI4 $\geq 5$ events/h $(n=6)$} & \multirow{2}{*}{$P^{*}$} \\
\hline & Mean \pm SD & Range & Median & Mean $\pm \mathrm{SD}$ & Range & Median & \\
\hline Total mass, kg & $101.8 \pm 18.3$ & $76.5-138.2$ & 102.2 & $117.6 \pm 13.1$ & $95.1-130.8$ & 119.4 & .03 \\
\hline Total fat mass, $\mathrm{kg}$ & $21.4 \pm 11.0$ & $6.8-44.7$ & 19.4 & $31.5 \pm 10.5$ & $18.8-42.6$ & 34.2 & .062 \\
\hline Total fat, $\%$ & $20.0 \pm 7.3$ & $8.8-34.1$ & 18.4 & $26.3 \pm 6.7$ & $16.8-34.3$ & 28.1 & .069 \\
\hline Total lean mass, $\mathrm{kg}$ & $76.3 \pm 9.5$ & $58.9-103.5$ & 77.5 & $81.7 \pm 6.5$ & $72.5-89.5$ & 82.5 & .11 \\
\hline Trunk fat mass, $\mathrm{kg}$ & $9.9 \pm 6.2$ & $2.2-25.3$ & 8.5 & $14.7 \pm 5.2$ & $8.4-19.8$ & 15.8 & .08 \\
\hline Trunk fat $\%$ of total fat mass & $43.9 \pm 6.8$ & $27.0-56.8$ & 44.5 & $46.3 \pm 2.6$ & $43.1-49.2$ & 46.1 & .12 \\
\hline Abdominal visceral fat mass, $\mathrm{kg} \dagger$ & $0.30 \pm 0.33$ & $0.00-1.3$ & 0.19 & $0.65 \pm 0.39$ & $0.22-1.1$ & 0.76 & .11 \\
\hline Visceral fat $\%$ of total fat mass $\dagger$ & $1.5 \pm 1.2$ & $0.01-4.8$ & 1.1 & $1.9 \pm 0.63$ & $1.2-2.8$ & 2.0 & .25 \\
\hline
\end{tabular}

quently underwent overnight polysomnography. An AHI of $\geq 10$ was found in $13(34 \%)$ of the high-risk players, compared with just one $(7 \%)$ in the low-risk group. In a follow-up analysis, ${ }^{5}$ these investigators reported that 10 additional players had an AHI of between 5 and 9.9 events/h, thereby increasing the estimated prevalence rate of SDB in this athletic cohort to nearly $50 \%$, if using a more traditional AHI threshold. More recently, Rice et al ${ }^{6}$ evaluated SDB among 137 active veteran NFL players using a homebased, unattended, portable sleep monitor. The prevalence of at least mild SDB (respiratory disturbance index $\geq 5$ ) was $19 \%$. Although linemen weighed more and had a greater body fat percentage, waist circumference and neck circumference compared with non-linemen, there was no difference in SDB prevalence estimates between these groups. However, the average body mass index across both studies was $>30$, a noteworthy finding given that increased body weight is perhaps the strongest predictor of SDB. ${ }^{15,16}$ Thus, despite presumably being highly conditioned, these young athletes are at significantly greater risk for SDB than community-dwelling men of similar age. ${ }^{7}$

The current study extends this line of inquiry in several meaningful ways. First, we have estimated the prevalence of SDB in a Division 1AA collegiate football team. Our observed prevalence of approximately $8 \%$ for those with at least mild SDB (AHI $\geq 5$ ) is lower than what has been reported previously in the professional ranks, but we feel it is quite significant considering that sleep screenings are not likely to be part of most physical exams for collegiate athletics programs. By 2015, the number of United States colleges and universities offering football will have increased to 773 (http://www.footballfoundation.org. Accessed August 20, 2015). Assuming that most teams maintain rosters of about 100 athletes, this equates to roughly 6,000 players who are living and competing with SDB. The clinical and public health importance of this is underscored by the fact that other studies ${ }^{17,18}$ have re- ported early signs of atherosclerosis (eg, endothelial dysfunction, increased carotid intima-media thickness, and arterial stiffening) among minimally symptomatic, middleage OSA subjects, suggesting that if left untreated, collegiate football players with SDB are likely to suffer from a greater cardiovascular disease burden after their athletic careers have ended. Perhaps of more immediate concern is the adverse impact sleep apnea has on attention and cognitive function. ${ }^{8}$ The potential implication is that players with SBD may experience detriments in academic as well as athletic performance. ${ }^{19}$

Second, emerging data suggest that proper sleep may play an important role in recovery from and possibly prevention of concussions. ${ }^{20}$ That said, our finding of an average total sleep time of $4 \mathrm{~h}$ is alarming. Although the short sleep duration may be attributable to extraneous factors (eg, wearing the PPG device and anxiety associated with preseason camp [see below]), existing data suggest ${ }^{21}$ that insufficient sleep and poor quality sleep in college students is more likely the rule, than the exception. Indeed, $35 \%$ of the players in this study reported a clinically important degree of daytime sleepiness.

Third, we found that the STOP-BANG questionnaire had low sensitivity to predict mild to severe SDB (50\%). By comparison, the sensitivity to detect positive cases increased to $75 \%$ when using the Epworth Sleepiness Scale. Our decision to choose the STOP-BANG was based on ease of use and the simple scoring system. Thus, it is more likely to be incorporated into a sports medicine setting. However, it appears that it may lack sufficient sensitivity in this cohort to avoid missing a large number of SDB cases. The reasons for this may be related to the fact that some of the elements of the STOP-BANG (1) could not be observed (eg, player may lack a bed partner to observe whether he stops breathing), (2) were constants (eg, all players were men $<50$ y old), and (3) were also contained in body composition measures (eg, body mass index and 
neck circumference). Clearly, our findings call into question the suitability for use of the STOP-BANG to identify positive cases in this demographic group.

Fourth, we employed state-of-the-art dual-energy x-ray absorptiometry imaging that allowed us to quantify total and regional fat with greater precision than previous reports. ${ }^{5,6}$ Consequently, we found higher regional (ie, trunk fat mass and abdominal visceral fat mas) and total body fat mass in players with oxygen desaturations above a threshold of 5 events/h, albeit most of these difference did not reach statistical significance. However, we cannot discount the possibility that we made a type- 2 error. Still, this observation is consistent with the notion that central adiposity increases OSA susceptibility by elevating mechanical loads on the upper airway. Also, adipose tissue is an abundant source of pro-inflammatory cytokines, such as TNF- $\alpha$, and IL-6. These are markedly elevated in central obesity $^{22,23}$ and their purported somnogenic activity ${ }^{24}$ may lead to a depression in upper airway neuromuscular control.

The above findings should be viewed in the context of several limitations. The sample size was small, thereby limiting our power to detect (1) group differences in the number of high-risk versus low-risk players who have clinical signs of SDB (eg, ESS $>10$, AHI/ODI $\geq 5$ events/h) or (2) differences in body composition parameters between those with and without SDB. Moreover, although ethnicity may affect body composition, the small sample precludes us from making meaningful comparisons between racial groups. Also, we used one night of PPG monitoring, and all tests were performed during preseason mini-camp. During this time, the players undergo intensive training, experience high levels of stress, and sleep in dormitory beds. These factors are likely to affect sleep habits and, in turn, ESS scores. Further, any direct comparisons of SDB prevalence rates between collegiate and professional football players must be interpreted with caution, since George et $\mathrm{al}^{14}$ relied on overnight polysomnography and Romem et $\mathrm{al}^{11}$ used a portable monitoring system with air flow measurements to diagnose OSA. Although prior investigations $^{11-13}$ have shown that PPG displays a sensitivity ranging from 80 to $98 \%$, we acknowledge that these studies were conducted in subjects who were referred for overnight sleep testing because of clinically suspected SDB.

Also, Romem et al ${ }^{11}$ failed to show a significant correlation between polysomnography-derived and PPG-based parameters for total sleep time, which they attributed, in part, to possible degradation of transmission signal from the PPG to the cellphone receiver at the bedside. Accordingly, studies designed to examine the validity and reliability of PPG in this younger, athletic cohort are warranted. Related to this point, because the PPG is a single-channel device, it does not meet American Academy of Sleep Medicine standards for home sleep studies. Therefore, it is not ac- ceptable for insurance reimbursement or prescriptive treatment. Alternatively, it may serve as a good indictor for further sleep disorder diagnostic studies for players with an AHI or ODI of $\geq 5$ events/h. Last, we did not assess physiological markers of cardiovascular disease burden, markers of athletic or academic performance, or cognitive function, so it is not possible to draw conclusions regarding the potential impact of SDB in these players. However, we feel that the above limitations are balanced by our unique sample and the clear advantage PPG monitoring has over laboratory sleep studies with regard to cost effectiveness, convenience, and noninvasiveness.

\section{Conclusions}

In summary, we found the estimated prevalence of SDB (AHI $\geq 5$ ) among a group of Division 1AA collegiate football players to be approximately $8 \%$. Players stratified as high-risk had a similar prevalence rate compared with low-risk players. Thirty-five percent of the sample reported a clinically important degree of daytime sleepiness. Moreover, our data suggest that players with greater oxygen desaturations have high total body and regional fat. Hence, our data emphasize the need to screen for sleep disorders, educate these athletes about the importance of sleep, and help facilitate the translation of this knowledge into practice.

\section{REFERENCES}

1. Patil SP, Schneider H, Schwartz AR, Smith PL. Adult obstructive sleep apnea: pathophysiology and diagnosis. Chest 2007;132(1):325337.

2. Peppard PE, Young T, Palta M, Skatrud J. Prospective study of the association between sleep-disordered breathing and hypertension. N Engl J Med 2000;342(19):1378-1384.

3. Gottlieb DJ, Yenokyan G, Newman AB, O'Connor GT, Punjabi NM, Quan SF, et al. Prospective study of obstructive sleep apnea and incident coronary heart disease and heart failure: the sleep heart health study. Circulation 2010;122(4):352-360.

4. Young T, Peppard PE, Gottlieb DJ. Epidemiology of obstructive sleep apnea: a population health perspective. Am J Respir Crit Care Med 2002;165(9):1217-1239.

5. George CF, Kab V, Levy AM. Increased prevalence of sleep-disordered breathing among professional football players. N Engl J Med 2003;348(4):367-368.

6. Rice TB, Dunn RE, Lincoln AE, Tucker AM, Vogel RA, Heyer RA, et al. Sleep-disordered breathing in the National Football League. Sleep 2010;33(6):819-824.

7. Bixler EO, Vgontzas AN, Ten Have T, Tyson K, Kales A. Effects of age on sleep apnea in men: I. Prevalence and severity. Am J Respir Crit Care Med 1998;157(1):144-148.

8. Gelir E, Başaran C, Bayrak S, Yağcioğlu S, Budak MT, Fırat H, et al. Electrophysiological assessment of the effects of obstructive sleep apnea on cognition. PloS One 2014;9(2):e90647.

9. Silva GE, Vana KD, Goodwin JL, Sherrill DL, Quan SF. Identification of patients with sleep disordered breathing: comparing the four-variable screening tool, STOP, STOP-Bang, and Epworth Sleepiness Scales. J Clin Sleep Med 2011;7(5):467-472. 


\section{Prevalence of SDB in College Football Players}

10. Johns MW. A new method for measuring daytime sleepiness: the Epworth sleepiness scale. Sleep 1991;14(6):540-545.

11. Romem A, Romem A, Koldobskiy D, Scharf SM. Diagnosis of obstructive sleep apnea using pulse oximeter derived photoplethysmographic signals. J Clin Sleep Med 2014;10(3):285-290.

12. Amir O, Barak-Shinar D, Henry A, Smart FW. Photoplethysmography as a single source for analysis of sleep-disordered breathing in patients with severe cardiovascular disease. J Sleep Res 2012;21(1): 94-100.

13. Barak-Shinar D, Amos Y, Bogan RK. Sleep disordered breathing analysis in a general population using standard pulse oximeter signals. Sleep Breath 2013;17(3):1109-1115.

14. George CF, Kab V, Kab P, Villa JJ, Levy AM. Sleep and breathing in professional football players. Sleep Med 2003;4(4):317-325.

15. Young T, Peppard PE, Taheri S. Excess weight and sleep-disordered breathing. J Appl Physiol 2005;99(4):1592-1599.

16. Young T, Shahar E, Nieto FJ, Redline S, Newman AB, Gottlieb DJ, et al. Predictors of sleep-disordered breathing in community-dwelling adults: the Sleep Heart Health Study. Arch Intern Med 2002; 162(8):893-900

17. Drager LF, Bortolotto LA, Lorenzi MC, Figueiredo AC, Krieger EM, Lorenzi-Filho G. Early signs of atherosclerosis in obstructive sleep apnea. Am J Respir Crit Care Med 2005;172(5):613-618.
18. Kohler M, Craig S, Nicoll D, Leeson P, Davies RJO, Stradling JR Endothelial Function and Arterial Stiffness in Minimally Symptomatic Obstructive Sleep Apnea. Am J Respir Crit Care Med 2008; 178(9):984-988.

19. Fullagar HH, Skorski S, Duffield R, Hammes D, Coutts AJ, Meyer T. Sleep and athletic performance: the effects of sleep loss on exercise performance, and physiological and cognitive responses to exercise. Sports Med 2015;45(2):161-186.

20. McClure DJ, Zuckerman SL, Kutscher SJ, Gregory AJ, Solomon GS. Baseline neurocognitive testing in sports-related concussions: the importance of a prior night's sleep. Am J Sports Med 2014;42(2): 472-478.

21. Lund HG, Reider BD, Whiting AB, Prichard JR. Sleep patterns and predictors of disturbed sleep in a large population of college students. J Adolesc Health 2010;46(2):124-132.

22. Arner P. Regional differences in protein production by human adipose tissue. Biochem Soc Trans 2001;29(Pt 2):72-75.

23. Dusserre E, Moulin P, Vidal H. Differences in mRNA expression of the proteins secreted by the adipocytes in human subcutaneous and visceral adipose tissues. Biochim Biophys Acta 2000;1500(1): 88-96.

24. Opp MR. Cytokines and sleep. Sleep Med Rev 2005;9(5):355-364.

This article is approved for Continuing Respiratory Care Education credit. For information and to obtain your CRCE

(free to AARC members) visit www.rcjournal.com

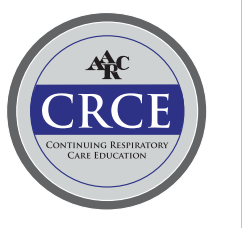

